\title{
OBJECT DETECTION USING REAL TIME ALGORITHM WITH FACE RECOGNITION
}

\author{
S.Kasthuri ${ }^{1}$, P.Ranjidha ${ }^{2}$, R.Saranya ${ }^{3}$ \\ ${ }^{1,2}$ M.E CSE -II ${ }^{\text {nd }}$, Year, ${ }^{3}$ Assistant Professor, Srinivasan Engineering College, Perambalur, Tamil Nadu, India
}

\begin{abstract}
A real and accurate object detection and Recognition technique is used to detect the real objects in the human face such as eye, nose etc... The face recognition is applicable to detect only the exact human face. But it does nose recognize the human face if there is any damage found in the face. To overcome these problem implement the Bayesian algorithm is used to detect the objects on human face like eye, nose etc... By using these algorithm the correct authorized user can be identified by comparing the current pixel values of the real object with the pixel values already stored in the database This system use C4 algorithm along with the MSRF (Multi Scale Random Field) because these methods are used to calculate only the neigbhouring pixel value by providing the fast rejection on the background image. Grayscale conversion from an RGB value results in the easy calculation of the pixel value. Pixel by pixel calculation is done and provides the secure authorization for the user against the hackers because the pixel value of an particular authorized person is entirely differ from the hackers, which can be get detected by SMAP (Sequential MAP)while handling transaction in an ATM Machine.
\end{abstract}

Keywords-Multiscale random field, multiscale random field

\section{INTRODUCTION}

Face recognition and face detection are used in various fields such as biometric authentication, authorization, and in many variety of applications .Certain aspects which is to be occurred while processing the images such as lighting, quality of an image, and also depends upon the pose and facial expression. In the earlier the facial recognition techniques can be subdivided into two major categories such as image preprocessing and artificial intelligence. The face recognition technique can be handled in two ways such as face identification and face verification. For calculating the verification perfomance it depends upon the verification rate (the way in the user granted access are to be get legitimate)along with the false accept rate .Face identification can be done by comparing existing image with the store images found in the database as the template value. In these face recognition techniques the face is to be get detected in an image and provide the template matching to localize the face. The eyes of different shapes are to be get identified on different facial images through luminance. Another important step to be followed along with the detection of eye is input data limitation which provides the size of the input image is $60 \mathrm{X} 80$ pixels or it provides the detection with an low accuracy either in an grayscale or digital image format. An Bayesian algorithm is implemented in the face recognition technique which is mainly used for secure authorization against the hackers. Because nowadays the hackers are able to hack the account with their stolen debit card. To avoid these problems Face recognition is applied along with the detection of objects found in the face. Eye is mainly used for handling the secured transaction.

\section{FACE DETECTION}

The following diagram shows the detection of objects in the face is to be pointed out.

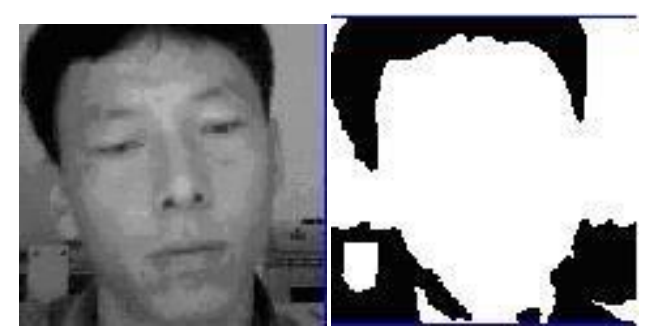

Fig 1 Face Detection

Face detection is mainly used for secured authorization process. From the capturing image the conversion of grayscale image is carried out for providing the easier calculation of pixel values for particular extracted part from the face such as eye ,nose, etc....Then the detection can be takes place by the contour information provided by the $\mathrm{c} 4$ framework which pays the way for early rejection of unwanted background while capturing an image by finely encoding the calculated neighboring pixel value. CENTRIST Visual descriptor is used along with the $\mathrm{C} 4$ framework which provides an detection not in an appropriate manner. 
Along with the use of an Bayesian algorithm the face can be detected exactly with an super resolution which may provide an secure authorization to the user by detecting the real objects at the current position and by comparing the detected objects with objects already stored in the database. for example if the nose gets damaged means the pixel values may get differ with pixels already stored in the database as a template value. In this proposed work eye is only used for authorization purpose because eye is used as the main detecting object which is used for secure authorization while handling transaction in ATM machine. The hackers not able to hack the user account because the pixel values may get mismatched. This is the appropriate way to secure account from the hackers. This is the suitable way for providing the transaction for the authorized user by calculating the pixel value of the authorized person by detecting the face

\section{SKIN COLOR SEGMENTATION}

The identification of skin region is found to be play an important role in the detection of eye of because it easily determine the appropriate fixed position o. Beginning with a color image, the first stage is to transform it to a skinlikelihood image.The eye by eliminating. This involves transforming every pixel from RGB representation to chroma representation and determining the likelihood value based on the equation given in the prhe false eye candidates. previous section. The skin-likelihood image will be a gray-scale image whose gray values represent the likelihood of the pixel belonging to skin. A sample color image and its resulting skinlikelihood image are shown in figure. The figure shows that the skin is differentiated into origina, skinlikelihood and segmented image. Finally the segmented part of the detected region is used to calculate the pixel value because the segmented part only used to calculate the pixel value along with the conversion of gray scale image ( 0 and 1$)$.
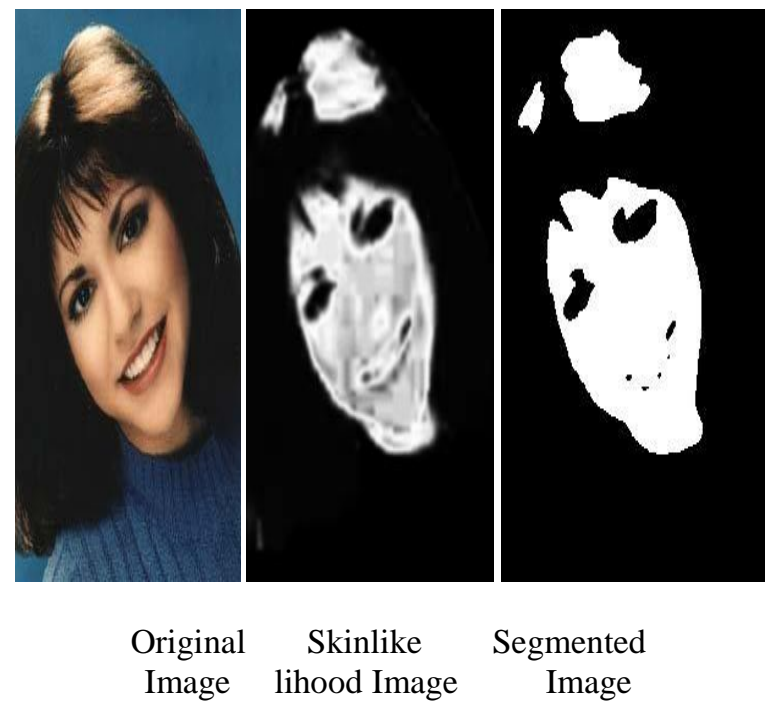

Fig 2 Skin Color Segmentation
The adaptive thresholding is based on the observation that stepping the threshold value down may intuitively increase the segmented region. However, the increase in segmented region will gradually decrease (as percentage of skin regions detected approaches $100 \%$ ), but will increase sharply when the threshold value is considerably too small that other non-skin regions get included. The threshold value at which the minimum increase in region size is observed while stepping down the threshold value will be the optimal threshold. In our program, the threshold value is decremented from 0.65 to 0.05 in steps of 0.1 . If the minimum increase occurs when the threshold value was changed from 0.45 to 0.35 , then the optimal threshold will be taken as 0.4.The process of identifying skin region is found to be important for identifying eyes which is to be used for the secure authorization. The skin color segmentation which is used for comparing the exact eye position by eliminating a huge number of false eye candidates. In this paper, we have used a combination of, C4, CENTRIST visual descriptor and Bayesian algorithm is used to increase the performance to detect the eye .The unwanted pixel values are to be carefully removed such as facial hairs, creases. A morphological binary image method is used for combining both the image which is already stored in the database with the existing skin image by using the AND operation. Thresholding is also used along with morphological process for the quick detection of eyes with minimum noise by removing the stray points.

\section{EYE DETECTION}

The process of eye gaze tracking, iris detection, video conferencing, faces recognition and face detection. Eye detection is mainly used in the face detection for handling the secure transaction against the hackers. Bayesian algorithm is mainly used for pixel by pixel calculation whereas $\mathrm{C} 4$ is just used for providing that the neighboring pixel value calculation. Even though the detection of eye can be used in the application such as hand free cursor which means that handicapped persons are able to use computers without the use of others by using fixed common values found along the middle position of the two eyes. The localization of eye can be achieved for comparing the facial expression in image along with the current facial position of the particular person. In eyes detection, convert an RGBface to binary face, because the pixel value is not accurately calculated by using the RGB value... Now, Then assume the face width as $\mathrm{W}$. Then the edge detection is another important task in eye detection. Then scan from the $\mathrm{W} / 4$ to $(\mathrm{W}-\mathrm{W} / 4)$ to find the middle position of the two eyes. The highest white continuous pixel along the height between the ranges is the middle position of the two eyes. The eye can be detected only from the sharp point of reflection while detecting the object in face recognition. Then the NTSC format is format is mainly used for separating the gray scale information which pays the way for easy calculation of pixel value by using the c4 along with the Bayesian algorithm. The finite number of eye candidate is to 
be removed and boundary of the eye can be detected through the morphological process by removal of noise. Eyes play an important role in the process of image normalization. The image normalization can be achieved by calculating the interocular distance between the middle positions of the two eyes. The detection of eye is to be shown in the figure

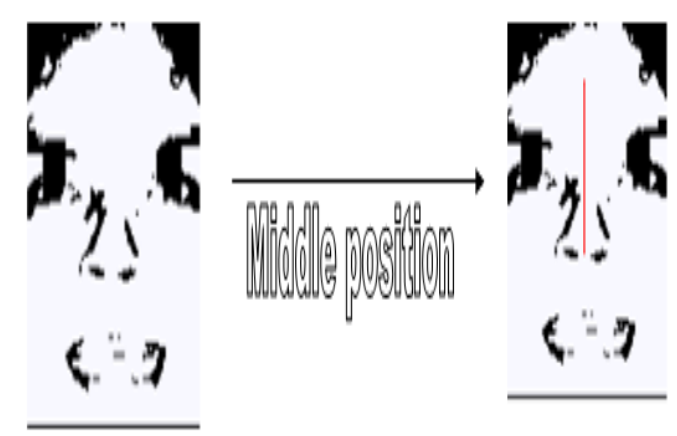

Fig 3 Pixel calculation in eye

\section{DATABASE VERIFICATION}

Database verification is mainly involves in comparing the pixel values which is stored as a default value with the current pixel values of the user by using the interpersonal and intrapersonal variations.

Intrapersonal variation: These are used to identify the common similarities found in the same individuals.

Interpersonal variation: These are used to identify the different similarities found in the same individuals.

\section{PROPOSED WORK}

The face is identified correctly even in an low resolution power which replaces SR-preprocessing. The Bayesian algorithm is used to find the objective function aims to preserve the locality between neighbor-hood in the reproducible kernel Hilbert space. In this method, the final kernel matrix is constructed by concatenating two individual kernel matrices (linear, polynomial) in the diagonal direction, and the (semi)positively definite properties are preserved for optimization.

\section{BACKGROUND OF THE ALGORITHM}

Bayesian algorithm which is used as an brute force speed for object detection. These algorithm are able to detect the window (size of $\mathrm{h} \mathrm{xw}$ ) on the stored input image versus the current window size of the authorized user), which is able to classifies the each detection window as the object of interest or background. During the detection of all possible windows then all the available topmost left corners form an regular grid step which can be allocated even to the larger value also. If the possible detected window size is found to be larger than (hxw) then they are scanned to the value as 0.8 until the image size is reduced smaller than (hxw).Finally Bayesian algorithm is implemented along with the combination of Sequential MAP which is able to convert the RGB color image into grayscale image with minimum resolution to calculate the pixel value easier which is merely used for secure authorization while handling transaction in ATM machine.

\section{ARCHITECTURE OVERVIEW}

This architectural diagram provides the overall pixel calculation of the face and how they are used for secured authorization purpose. Face is divided into various segments such as eyes, nose mouth etc. then calculate the pixel value for each segmented region.

The process of segmenting the face involves various process such as eye, nose, etc. Particularly it involves in segmenting the eye and removes the eye brow from both left and right eye performing the pixel calculation and stored the calculated value as template value. while get authorizing during handling transaction it checks with the existing eye along with the stored template value and provide the secure transaction to particular authorized user against from the hackers.

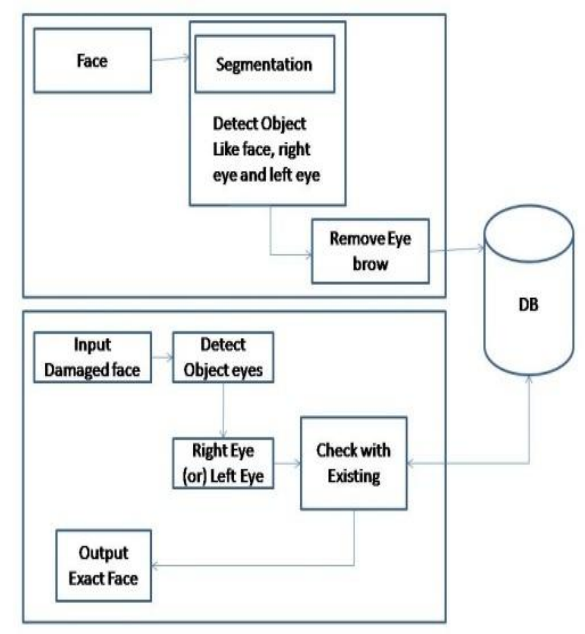

Fig 4 Architectural diagram

\section{CONCLUSIONS}

By using the Bayesian algorithm along with the 4 frameworks and centrist visual descriptor provides the pixel by pixel calculation and provide the secure transaction to the particular user against the hackers in an efficient way.

\section{ACKNOWLEDGEMENTS}

We would like to thank our college Srinivasan Engineering College, our HOD Mrs. S.Jeyanthi , and our guide R.saranya and other staff for their continous support and for their helpful comments on the earlier drafts of this paper. 


\section{REFERENCES}

[1]. S. Maji and A. C. Berg, "Max-margin additive classifiers for detection," in Proc. IEEE Int'l Conf. on Computer Vision, 2009, pp. 40-47.

[2]. W. R. Schwartz, A. Kembhavi, D. Harwood, and L. S. Davis, "Human detection using partial least squares analysis," in Proc. IEEE Int'l Conf. on Computer Vision, 2009.

[3]. P. Doll'ar, Z. Tu, P. Perona, and S. Belongie, "Integral channel features," in Proc. British Machine Vision Conference, 2009.

[4]. B. Leibe, E. Seemann, and B. Schiele, "Pedestrian detection in crowded scenes," in Proc. IEEE Int'l Conf. on Computer Vision and Pattern Recognition, vol. I, 2005, pp. 878-885.

[5]. J. Wu, W.-C. Tan, and J. M. Rehg, "Efficient and effective visual codebook generation using additive kernels," Journal of Machine Learning Research, vol. 12, pp. $3097-$ 3118, Nov 2011

[6]. S. Paisitkriangkrai, C. Shen, and J. Zhang, "Incremental training of a detector using online sparse eigen decomposition," IEEE Trans. on Image Processing, vol. 20, no. 1, pp. 213-226, 2011

[7]. J. Gall, A. Yao, N. Razavi, L. V. Gool, and V. Lempitsky, "Hough forests for object detection, tracking, and action recognition," IEEE Trans. on Pattern Analysis and Machine Intelligence, vol. 33, no. 11, pp. 2188-2202, 2011.

[8]. C. Wojek, S. Walk, and B. Schiele, "Multi-cue onboard pedestrian detection," in Proc. IEEE Int'l Conf. on Computer Vision and Pattern Recognition, 2009.

[9]. R. Benenson, M. Mathias, R. Timofte, and L. V. Gool, "Pedestrian detection at 100 frames per second," in Proc. IEEE Int'l Conf. on Computer Vision and Pattern Recognition, 2012, pp. 2903-2910.

[10]. C. H. Lampert, M. B. Blaschko, and T. Hofmann, "Efficient subwindow search: A branch and bound framework for object localization," IEEE Trans. on Pattern Analysis and Machine Intelligence, vol. 31, no. 12, pp. 2129-2142, 2009. 